\title{
User Requirements Elicitation of Stereoscopic 3D Video Interaction
}

\author{
Haiyue Yuan, Janko Ćalić, Anil Fernando, Ahmet Kondoz \\ I-Lab, Multimedia Communication Research \\ Centre for Vision, Speech and Signal Processing \\ University of Surrey, United Kingdom \\ Email: h.yuan, c.calic,w.fernando, a.kondoz@surrey.ac.uk
}

\begin{abstract}
The recent development of three dimensional (3D) display technologies has resulted in a proliferation of 3D video production and broadcasting, attracting a lot of research into capture, compression and delivery of stereoscopic content. However, the predominant design practice of interactions with $3 D$ video content has failed to address its differences and possibilities in comparison the existing $2 \mathrm{D}$ video interactions. This paper presents a study of user requirements related to interaction with the stereoscopic 3D video. The study suggests that the change of view, zoom in/out, dynamic video browsing and textual information are the most relevant interactions with stereoscopic 3D video. In addition, we identified a strong demand for object selection that resulted in a follow-up study of user preferences in 3D selection using virtual-hand and ray-casting metaphors. These results indicate that interaction modality affects users' decision of object selection in terms of chosen location in 3D, while user attitudes do not have significant impact. Furthermore, the ray-casting based interaction using Wiimote can outperform the volume-based interaction technique using mouse and keyboard for object positioning accuracy.
\end{abstract}

Keywords-3D television(3DTV); 3D stereoscopic; 3D video interaction; User requirement; Interactive functionality; Interaction modality

\section{INTRODUCTION AND RELATED WORK}

With the recent development of 3D stereoscopic display technology, 3D movies and 3D TV programmes are becoming a commonplace in our everyday lives. The launch of a number of broadcasted 3D channels, such as Sky 3D and BBC HD, TV viewers can immerse into 3D experience in their own living room. There has been a significant amount of ongoing related research into 3D content capture, production and delivery. However, to the best of our knowledge, there has been very little research towards meaningful user interaction with real $3 \mathrm{D}$ video content.

The aim of our research is to study user practices and propose technical solutions and design guidelines to develop intuitive interaction for 3D video content. In this paper, we focus on the requirements study from users' perspective. We starts from eliciting user requirements of stereoscopic 3D video interaction emphasizing on the interactive functionalities and interaction modalities with the potential challenges. As the identification of strong demand for object selection, we then present a user preference study that investigate the impact of user attitudes, interaction modalities, depth profiles, and dominant eye on object selection in 3D.

There have been a number of studies that introduced advanced interactive 2D video user interfaces, facilitating intuitive interaction with video content. Two interactive video players, DRAGON (DRAGable Object Navigation) [1] and DimP (Direct Manipulation Player) [2] offer direct object manipulation of a video scene. Here, the user can browse the video by selecting and dragging an object in the scene instead of using the timeline slide. In addition, other features such as motion trajectories and annotations were used by Goldman [3], providing more categories for direct interaction with video content.

Thanks to the development of stereoscopic display technology, 3D video is able to offer an immersive experience to wide audiences. However, compared with the plethora of research for 2D video interaction, there is very little research focusing on interacting with 3D video content. A lot of research has been dedicated to develop intuitive interaction modalities for $3 \mathrm{D}$ stereoscopic $\mathrm{CG}$ content in virtual reality and $3 \mathrm{D}$ user interface communities. Park et al. [4] present an interactive 3DTV interface with an intelligent remote controller, which enables the user to change the viewpoint from the controller according to visual attention model. Ki and Kwon [5] developed a gaze-based interaction application, which is based on the calculation of degree of eye rotation and pupil center distance to interact with 3D content. Furthermore, Steincke et al. [6] introduced the concept of interscopic interaction which means the visualization of $3 \mathrm{D}$ data is using stereoscopic techniques whereas the user interaction is performed via 2D graphical user interfaces. A recent research output of the MUSCADE project [7] introduced a Samsung smart mobile phone with an intelligent remoter controller to switch view between $3 \mathrm{D}$ video content and $3 \mathrm{D}$ CG content.

\section{USER REQUIREMENT STUDY}

The aim is to elicit the user requirement and user preference for interacting with 3D stereoscopic TV in terms of interactive functionalities and interaction modalities. Interview is commonly used as a method to explore specific issue [8] in user requirement analysis. Semi-structured interview was implemented in this study to identify the requirements. 


\section{A. Participants and Procedure}

This study included a total number of 10 participants. All participants are male and aged from 24 to 30 years old. They are from the same research center and studying or working in 3D video related research areas. Each participant has previous experience of watching 3D video.

The literature review and current practice of using 2D $\mathrm{TV} / \mathrm{video}$ and 3D TV/video were used as the base to form the structure of the interview. It consists of four parts: 1) To gather background information for each participant. 2) To learn about the current usage of interactive service or applications for 2D video content. 3) To identify the user requirements for interactive functionalities. 4) To elicit the requirements for user interface to facilitate intuitive interactions. All the interviews were recorded using either audio recorder or video recorder, and transcribed entirely afterwards. The categorization scheme was used to analyze the transcripts.

\section{B. Results}

1) Interactive Functionality Requirements: The result indicated that everything that works in $2 \mathrm{D}$ video can be applied for $3 \mathrm{D}$ video. The analysis of transcripts focused on the interaction functionalities, which are tailored for 3D video content but not necessary for 2D video content.

Changing the Angle of View: One of the expected functionalities for the future 3D interactive video system was changing the angle of view. However, there was a differentiation of opinions between participants regarding the way of achieving this objective. One proposition was that the user can select an object or a region then manipulate it to change the view point of the scene accordingly. Another proposition was to track viewer's head to change the angle of view. However consider the characteristics of 3D video, it is more practical to implement this functionality using 3D multi-view video rather than 3D stereoscopic video. Speaking of content requirement, movie and sports program were considered as the main video content types that this interactive functionality can fit in.

Zoom in/out: Be able to zoom in/out the 3D video content was one demanding requirement. It was expected to allow user to firstly select an object and then change the depth of the chosen object to make the illusion like pull the object close to audience, while keeping other objects in the scene at the original depth and original scale. The opposite recommendation was to zoom in the whole scene while all the objects in the scene should be scaled accordingly to keep the relative scale. There was no conclusive agreement of which way is more appropriate, it is a matter of user personalized choice. The possible solution might be providing compatible zoom in/out which can satisfy both requirements. The potential challenge of this issue in future work is to investigate the user preference of depth sensitivity, which can facilitate zoom in/out functionality and also improve user experience. The demanding video contents for this functionality were sports program, national geographical program, and documentary program were most in demand.

Textual information: Textual information based interaction allows the user to select an object in the scene to obtain corresponding information of the chosen object, which could be displayed in the format of text on the screen. The potential challenge for the textual information based interaction is to define where the text should be displayed, and how the text is displayed in 3D without distraction. Participants would like to use this interaction to access information of interested object in documentary program, to get knowledge of the footballer while watching a football game, or to obtain information of a actor/actress in a movie.

Dynamic video browsing: As a concept of select and drag an object in the scene to browse the video instead of time-line slide inspired by direct manipulation video player [2], [3], it can be adapted for 3D video content. The most interesting part for this interaction is to allow the user browse the video in three dimensions. However the concern was that the applicability mainly depends on the video content. It was not necessary to have this function for most of the programs, but only for application like video analysis such as high speed collision of objects, sports analysis, and surveillance analysis.

2) User Interface Requirements: The objective for this part is to find out the user preference of interaction modalities that can support 3D video interaction that proposed in previous stage. The dominant candidate was the hand gesture, however the concern of using hand gesture was critical. It mainly because: 1) The hand gesture might lack of accuracy in the case of selecting an object 2) Deal with the chaos caused by involuntary movement 3) Design an effective system for multiple users 4) Implement privacy control. Consider the above concerns, the alternatives were various such as small device with touch pad, virtual laser pointer, and digitalized glove. Although there was no conclusive result of user interface, the common agreement was that the user interface should merge the reality and virtual environment to offer immersive experience.

In addition, during the interview, it is not surprising to find that selection was frequently used as the first step for above proposed 3D video interaction. This is consistent with the findings from previous literatures, which indicate that selection is one of the essential building blocks of all interactive virtual environment systems. It is a process of identifying particular objects, which are the targets for the subsequent actions.

\section{User Preference of ObJect Selection In 3D}

According to the findings from user requirement analysis, the conclusive agreement among all participants was that selection can be considered as the fundamental requirement for proposed 3D video interaction. The following user study is 
to explore related issue and give recommendations for object selection in 3D. Selection has been extensively addressed in previous literatures. Most of the selection techniques are variations of the following two main classes: volume-based selection and ray-based selection [9], [10], [11]. Volumebased selection uses the virtual hand/cursor and cone selection to select an object, where requires intersection or collision detection between the virtual hand and the $3 \mathrm{D}$ object. Ray-based selection cast a virtual ray into the virtual world to select an object, which is hit by the virtual ray. We implemented both volume-based selection and ray-based selection techniques in this study in order to investigate the impact of different interaction modality on the user preference of 3D selective position.

\section{A. Experimental Setup}

In this part, we used 3D stereoscopic CG/animated content to conduct the experiment as it requires a controlled environment, which 3D stereoscopic video content could not offer. Our intention was to learn user behavior from this experiment and generate results of user preference of object selection in $3 \mathrm{D}$, which can be transferable benefits for the 3D stereoscopic video interaction in our future work. The experiment was performed on a 46" JVC stereoscopic display with passive polarization glasses (Model number GD-463D10). The resolution of the display is 1920x1080 and the recommended viewing distance is 2 meters from the screen. The supported format for stereoscopic content is left and right side-by-side representation. We used mouse, keyboard, two Wiimotes with motion plus and a Wii sensor bar in the experiments. We produced and rendered the stereoscopic 3D content using OGRE (Open Source 3D Graphics Engine). Figure 1 (a) presents the set up during the experiment using Wiimote.

\section{B. Participants and Procedure}

There were 15 participants in this experiment. They are all research students in the same research lab. Participants were aged 21 to 28, and contained 1 female and 14 male. All the participants have previous experience of watching $3 \mathrm{D}$ stereoscopic video and playing 3D game. 5 of them are left eye dominant, and 10 of them are right eye dominant. Before conducting the experiment, each participant took a stereo acuity test, and all participants had accepted stereo perception.

The whole experiment consists of two parts. We implemented volume based selection technique as a virtual cursor metaphor in part 1 . In part 2, we adopted ray-based selection technique to design an prototype of virtual laser pointer, which combined Wiimote, Wii motion plus, and Wii sensor bar.

Each part contained 2 sets. For the first set, each participant was asked to take time to choose one object which he/she like the most, and then put the selected object into the destination. For the second set, each participant was required to do the same task as quick as possible. For each set, the participant needed to finish the selection task with 20 different depth profiles each trial for 3 trials. Therefore each participant completed the task for $3 \times 20 \times 2 \times 2$ trials for the whole experiment. It took around 30 minutes to complete each part of the experiment, and one hour for the whole experiment.

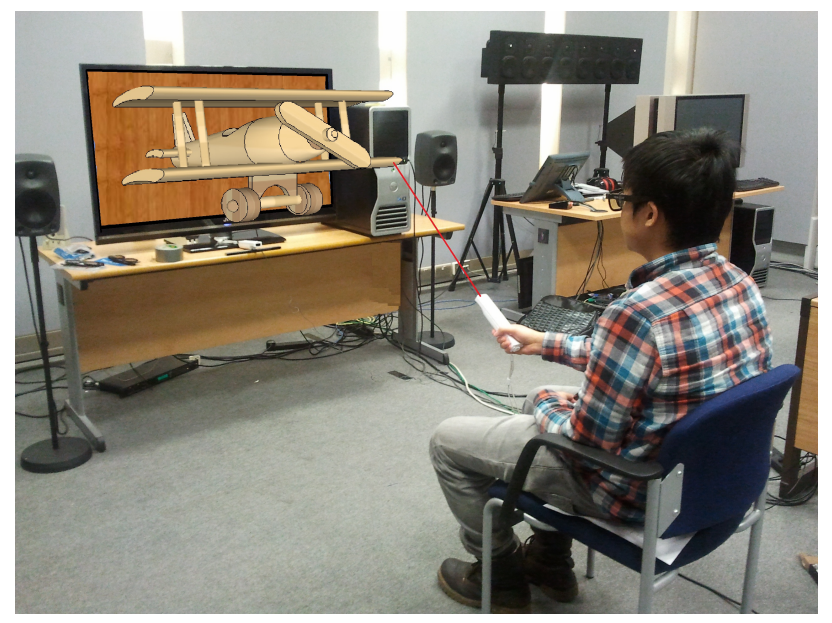

(a)

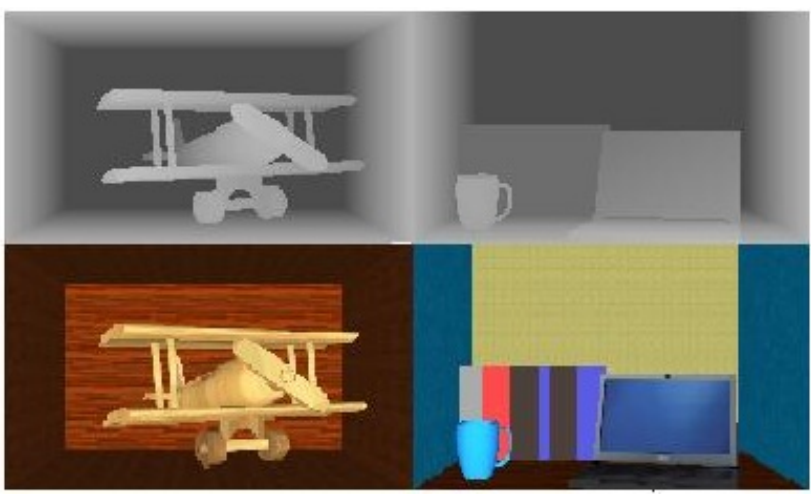

(b)

Figure 1. (a) Experimental setup used in the user preference study with Wiimote (b) Two examples of the 3D scenes with their depth maps used in user preference study

The display was divided into 9 sub screens. For each trial, 1 object was random allocated to its respective sub screen within each depth profile. Depth profile was used to simulate the different 3D scene. Figure 1(b) gives two examples of depth profile with associated depth map. The reason we created different depth profile was twofolds, one was attempting to find out the relationship between user preferred selective position and associated depth profiles, another one was to provide distractive background to make the task more realistic. 


\section{Experimental Results}

The results in this section present the impact of different parameters on the user preference of object selection in 3D.

1) User Attitude Impact: The participant was asked to choose the object in two different attitude, one was to Take Time to choose the object which he/she like the most and then put it into the destination, another attitude was to choose the object A.S.A.P (as soon as possible) and then put it into the destination. ANOVA (Analysis of Variance) was used to analyze the statistic difference between two attitudes regarding the task completed time and task completed accuracy respectively. How far the placed object away from the selected destination was used to indicate the accuracy. The smaller the distance is, the higher the accuracy is. ANOVA showed a significant main effect $(F 1,1783=101.7$, $\mathrm{p}=.000$, see Table I) of user attitude on the task completed time. It is not surprising that participant spent about one more seconds in average to completed the task in Take Time attitude than in A.S.A.P attitude. For the accuracy, there was no significant difference between two groups ( $\mathrm{F} 1,1783=.99$, $\mathrm{p}=.319$ ), which indicated that the user attitude did not have significant impact on the accuracy of completing the task.

Table I

IMPACT OF USER ATTITUDES TO TASK COMPLETION TIME AND ACCURACY

\begin{tabular}{|l|l|l|}
\hline & $\begin{array}{l}\text { Task Completed } \\
\text { Time }\end{array}$ & Accuracy \\
\hline Attitude & Mean (std) & Mean (std) \\
\hline Take Time & $3.73 \mathrm{~s}(3.00)$ & $.727(1.862)$ \\
\hline A.S.A.P & $2.61 \mathrm{~s}(1.36)$ & $.645(1.637)$ \\
\hline ANOVA Test & $\begin{array}{l}\mathrm{F} 1,1784=101.7, \\
\mathrm{p}=.00\end{array}$ & $\mathrm{~F} 1,1784=.99, \mathrm{p}=.319$ \\
\hline
\end{tabular}

In addition, we investigated the impact of user attitude towards the matter of where the user wants to select the object in both 2 dimension and third dimension. The chosen rate of each sub screen was indicated by the percentage of chosen objects, which was the number of chosen objects divided by the total number of objects allocated in this sub screen. The corresponding distribution of object chosen percentage across sub screens is depicted in Figure 2 (a). Sub screen 4 , and sub screen 5 had highest percentage for both user attitude scenarios. Sub screen 2, sub screen 6 , and sub screen 7 had around 10 percent of chosen rate. In addition, we did a pairwise correlation test between two groups. Significant correlation between two groups $(r=.9483$, $\mathrm{p}=.0001$ ) indicated that the user attitude did not affect user choices of object selection in 2D domain.

Furthermore, we took a look at the user preference of object selection in third dimension. We clustered the position of objects in third dimension into three categories: near, middle and far. We measured the percentage of chosen objects against all the objects that are in the same depth cluster (please see Figure 3 (a)). For both scenarios, par-
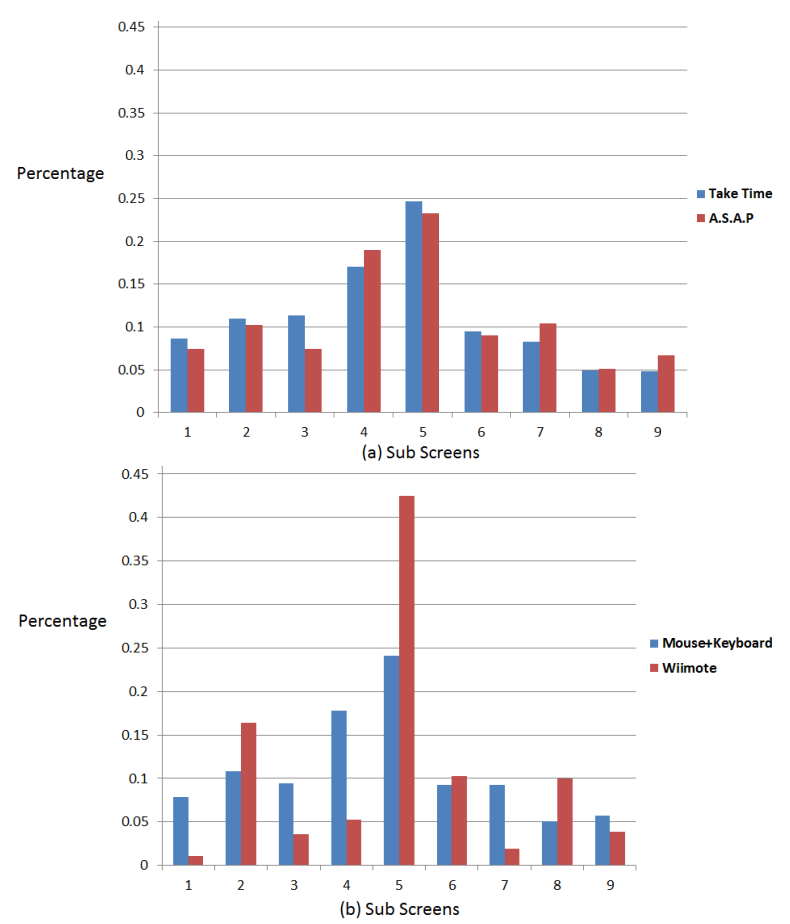

Figure 2. Percentage of chosen objects across sub-screen: a) User attitudes, b) Interaction modalities

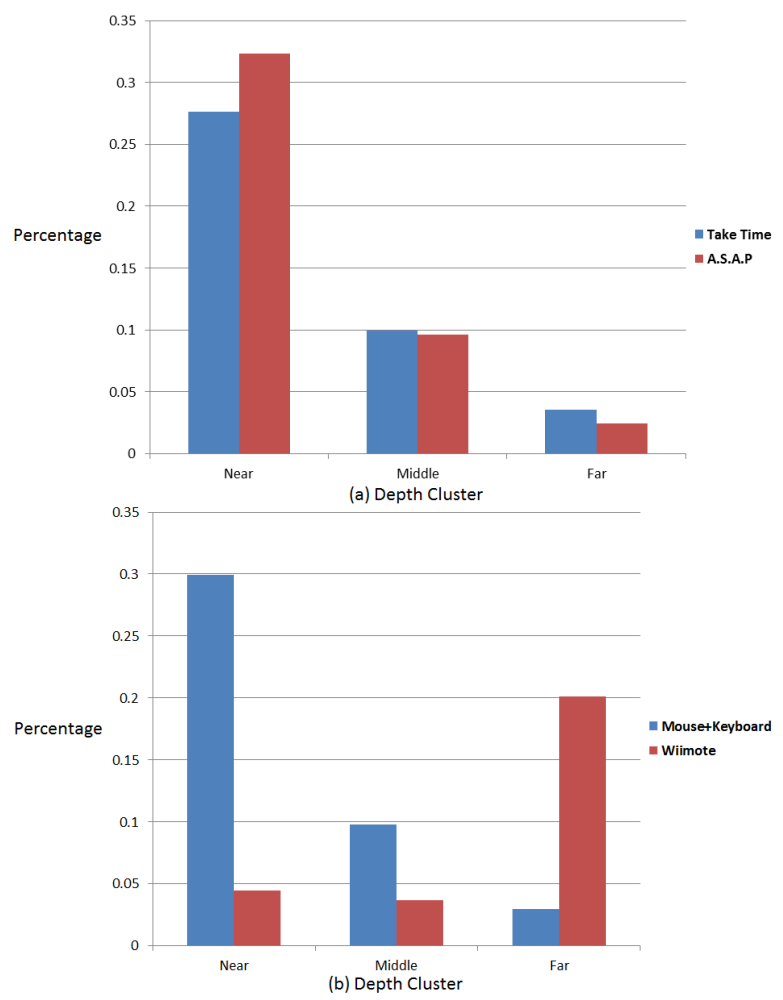

Figure 3. Percentage of chosen objects in third dimension: a) User attitudes, b) Interaction modalities 
ticipants preferred objects in front. The pairwise correlation test indicated the significant correlation between two groups $(\mathrm{r}=.9996, \mathrm{p}=.0017)$. Therefore user attitude did not have effect on the user preference of object selection in third dimension. Above analysis was based on volume selection interaction modality using mouse and keyboard. Similar results have been found for ray casting selection interaction modality using Wiimote.

2) Interaction Modality Impact: In this part, two interaction modalities were used to find out how do they affect user preference of 3D object selection. The dependent variable was task completed time and accuracy respectively, the independent variable was interaction modality, which contain Mouse+Keyboard and Wiimote two categories. ANOVA indicated no significant difference $(\mathrm{F} 1,1775=.07, \mathrm{p}=.7891$, see Table II) of task completed time between two modalities. For

Table II

IMPACT OF THE INTERACTION MODALITY TO THE TASK COMPLETION TIME AND ITS ACCURACY

\begin{tabular}{|l|l|l|}
\hline & $\begin{array}{l}\text { Task Completed } \\
\text { Time }\end{array}$ & Accuracy \\
\hline Modality & Mean (std) & Mean (std) \\
\hline Mouse+Keyboard & $3.17 \mathrm{~s}(2.41)$ & $.69(1.75)$ \\
\hline Wiimote & $3.22 \mathrm{~s}(2.22)$ & $.22(.61)$ \\
\hline ANOVA Test & $\mathrm{F} 1,1775=.07, \mathrm{p}=.79$ & $\begin{array}{l}\mathrm{F} 1,1775=17.61, \\
\mathrm{p}=.00\end{array}$ \\
\hline
\end{tabular}

the accuracy analysis, significant difference $(F 1,1775=17.61$, $\mathrm{p}=.000$ ) between two interaction modalities suggested that using Wiimote can offer higher accuracy of object positioning.

The comparison of object chosen rate in 2D between two interaction modalities across 9 sub screens is shown in Figure 2 (b). The correlation analysis found correlation between two interaction modalities $(\mathrm{r}=.7523, \mathrm{p}=.01)$ Although it was not highly correlated, sub screen 5 had the highest chosen rate for both scenarios and sub screen 2 and sub screen 6 had similar chosen rate.

The analysis of user preference of object selection in third dimension revealed that participant was more willing to choose further objects using Wiimote (see Figure 3 (b)). No significant correlation have been found between two modalities in this case $(\mathrm{r}=-.664, \mathrm{p}=.5373)$. The reason of such bias of object selection in third dimension was because of the interaction techniques. The informal post experiment interview also backed up this result. It was easier to use laser pointer like metaphor to reach anywhere in the scene. The interaction modality had significant impact on the user preference of object selection in third dimension, and less impact on the user preference of object selection in 2D.

3) Depth Profile Impact: There were 20 different depth profiles in this study, we conducted ANOVA test across different groups (user attitude group and interaction modality group) to investigate the relationship between depth profiles in terms of task completed time and accuracy respectively.
The dependent variable was task completed time and accuracy respectively, the independent variable was depth profile. As seen from Table III, there was no significant difference within depth profiles between different user attitudes, and between different interaction modalities. In addition, we

Table III

ANOVA TEST ACROSS DEPTH PROFILES

\begin{tabular}{|l|l|l|}
\hline & $\begin{array}{l}\text { Task Completed } \\
\text { Time }\end{array}$ & Accuracy \\
\hline Attitude & F19,1784(p) & F19,1784(p) \\
\hline Take Time & $1.52(.08)$ & $.67(.849)$ \\
A.S.A.P & $1.22(.2337)$ & $.7(.8198)$ \\
\hline Modality & F19,1775(p) & F19,1775(p) \\
\hline Mouse+Keyboard & $1.25(.2071)$ & $.71(.807)$ \\
Wiimote & $.89(.595)$ & $0.82(.679)$ \\
\hline
\end{tabular}

compared the correlation of object chosen rate each profile for 20 different depth profiles across different groups. For the majority of the depth profiles, participants had similar preference of object chosen rate across 9 sub screens no matter they take time to select the object or select the object as soon as possible. Only few significant correlation has been found for different interaction modalities. Numbers in bold in Table IV indicates significant correlation between groups for each corresponding depth profile. The results indicated

Table IV

CORRELATION TEST FOR EACH DEPTH PROFILE

\begin{tabular}{|c|c|c|c|c|c|}
\hline & Attitude & Modalities & & Attitude & Modalities \\
\hline DP & $\mathbf{r}(\mathbf{p})$ & $\mathbf{r}(\mathbf{p})$ & DP & $\mathbf{r}(\mathbf{p})$ & $\mathbf{r}(\mathbf{p})$ \\
\hline 1 & $.89(.00)$ & $.96(.00)$ & 11 & $.97(.00)$ & $0(1.0)$ \\
\hline 2 & $.97(.00)$ & $.98(.00)$ & 12 & $.99(.00)$ & $.10(.79)$ \\
\hline 3 & $.68(.04)$ & $-.39(.29)$ & 13 & $.20(.61)$ & $-.59(.09)$ \\
\hline 4 & $.64(.06)$ & $.48(.19)$ & 14 & $.86(.00)$ & $.78(.01)$ \\
\hline 5 & $.44(.24)$ & $-.18(.64)$ & 15 & $.74(.02)$ & $.34(.37)$ \\
\hline 6 & $.42(.26)$ & $0(1.00)$ & 16 & $.96(.00)$ & $.57(.11)$ \\
\hline 7 & $.96(.00)$ & $.29(.45)$ & 17 & $.39(.30)$ & $-.20(.61)$ \\
\hline 8 & $.46(.21)$ & $.58(.10)$ & 18 & $.57(.11)$ & $.66(.05)$ \\
\hline 9 & $.89(.00)$ & $.87(.00)$ & 19 & $.95(.00)$ & $.94(.00)$ \\
\hline 10 & $.85(.00)$ & $.32(.40)$ & 20 & $.72(.03)$ & $.14(.72)$ \\
\hline
\end{tabular}

DP stands for Depth Profile

that for different depth profiles, user attitudes had less impact than interaction modalities on the user preference of object selection. This is consistent with the previous findings from III-C1 and III-C2.

4) Dominant Eye Impact: In this part, we are looking at the impact of the dominant eye on the user preference of object selection. We expected a significant difference of user choices between left dominant eye participants and right dominant eye participants. The dependent variable was the relative horizontal distance between chosen object and center of the screen, where minus distance indicated that the object is located at the left side of the center and vice versa. The independent variable was dominant eye, where left eye dominant was indicated by dummy variable 0 and right eye dominant was indicated by dummy variable 1 . 
A robust linear regression test has been implemented, the result $(\mathrm{t}=4.17, \mathrm{p}=.0000, \mathrm{~F}=17.52, \mathrm{p}=.0000)$ suggests a significant difference between dominant eyes.

$$
\text { Distance }=-0.669+0.586 * E y e+c
$$

As given in the Eq. 1, if the participant is left eye dominant (i.e. Eye $=0$ ), the relative horizontal distance is -0.669 . On the contrary, if the participant is right eye dominant (i.e. Eye $=1$ ), the relative horizontal distance is -0.083 . The results indicated that participants with left dominant eye would choose the object more close to the left hand side than the participants with right dominant eye.

\section{CONCLUSion AND Future Work}

This study presents user studies that focus on user requirement of 3D video interaction, and user preference of object selection in 3D. The results as well as design recommendations are listed below.

- Change angle of view, textual information based interaction, zoom in/out, and dynamic video browsing are the interactive functionalities that can facilitate intuitive interaction with $3 \mathrm{D}$ video content. Object selection should be considered as the fundamental requirement for 3D video interaction.

- Users have consistent behavior of object selection over different user attitude while using the same interaction modality.

- Users have significant different preference of object selection especially in third dimension using different interaction modalities.

- The choice of where to select object in third dimension significantly depends on interaction modality.

- The area around the center of the screen has the highest object chosen rate regardless of user attitudes, interaction modalities across depth profiles.

- The virtual laser pointer based on ray-casting selection technique using Wiimote can offer higher accuracy of object positioning compare with volume-based selection using mouse and keyboard

- The user with left dominant eye prefer selecting the object more relatively close to left side of the display.

To continue this study, we propose the future work in two domains. One is dedicated to investigation of the methodology to achieve proposed interaction with $3 \mathrm{D}$ video content. The second one is to design experiments to quantify user performance of using different interaction modalities to complete interaction tasks, as well as investigate the impact of depth on the user experience of interacting with $3 \mathrm{D}$ video content. We hope our attempts to learn intuitive interaction with $3 \mathrm{D}$ video content from users perspective can provide understanding and guidelines, and bring interests to the research community.

\section{ACKNOWLEDGMENT}

This work was partly supported by the European Commission FP7 program, under the MUSCADE Integrated Project.

\section{REFERENCES}

[1] T. Karrer and et all, "Dragon: a direct manipulation interface for frame-accurate in-scene video navigation," in Proceedings of the twenty-sixth annual SIGCHI conference on Human factors in computing systems, ser. CHI '08. New York, NY, USA: ACM, 2008, pp. 247-250. [Online]. Available: http://doi.acm.org/10.1145/1357054.1357097

[2] P. Dragicevic and et al, "Video browsing by direct manipulation," in Proceedings of the twenty-sixth annual SIGCHI conference on Human factors in computing systems, ser. CHI '08. New York, NY, USA: ACM, 2008, pp. 237-246. [Online]. Available: http://doi.acm.org/10.1145/1357054.1357096

[3] D. Goldman and et al, "Video object annotation, navigation, and composition," in Proceedings of the 21st annual ACM symposium on User interface software and technology, ser. UIST '08. New York, NY, USA: ACM, 2008, pp. 3-12. [Online]. Available: http://doi.acm.org/10.1145/1449715.1449719

[4] M.-C. P., S. K. K., and J.-Y. S., "3d tv interface by an intelligent remote controller," in 3DTV Conference, 2007, may 2007, pp. $1-4$.

[5] J. K. and Y.-M. K., "3d gaze estimation and interaction," in 3DTV Conference: The True Vision - Capture, Transmission and Display of $3 D$ Video, 2008, may 2008, pp. $373-376$.

[6] F. Stenicke and et al, "Interscopic user interface concepts for fish tank virtual reality systems," in Virtual Reality Conference, 2007. VR '07. IEEE, march 2007, pp. $27-34$.

[7] "MUSCADE - Multimedia Scalable 3D for Europe, http://www.muscade.eu/."

[8] Y. Rogers, H. Sharp, and J. Preece, Interaction Design: Beyond Human-Computer Interaction. John Wiley and Sons Ltd, 2002.

[9] A. Steed, "Towards a general model for selection in virtual environments," in 3D User Interfaces, 2006. 3DUI 2006. IEEE Symposium on, march 2006, pp. 103 -110 .

[10] R. Teather and W. Stuerzlinger, "Pointing at 3d targets in a stereo head-tracked virtual environment," in $3 D$ User Interfaces (3DUI), 2011 IEEE Symposium on, march 2011, pp. $87-94$.

[11] D. A. Bowman, "Interaction techniques for common tasks in immersive virtual environments: design, evaluation, and application," Ph.D. dissertation, Atlanta, GA, USA, 1999, aAI9953819. 